\title{
Peter Hänni: Rosas Blut (1)
}

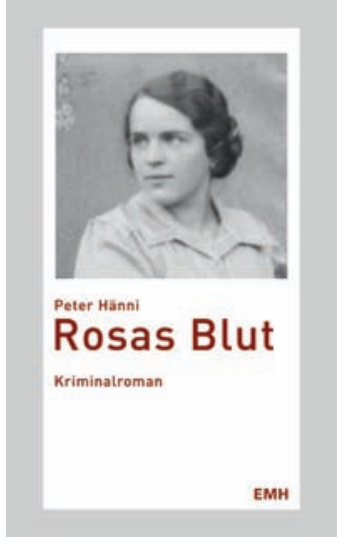

\section{Peter Hänni}

\section{Rosas Blut}

EMH Schweizerischer Ärzteverlag

Kriminalroman

2008. 192 Seiten. Broschiert.

Fr. $21.50 / € 14.80$

exkl. Versand

ISBN 978-3-0375-033-6

Die Plätze in dem kleinen Jet waren zu ungefähr zwei Drittel belegt. Luca Clemente und sein Vater saßen links vom Mittelgang, unmittelbar vor dem Flügel. Durch das Fenster, an dessen Rand sich ein trüber Rahmen aus Kondenswasser gebildet hatte, betrachteten sie die mächtigen, im Sonnenlicht gleißenden Alpen, die sie von Norden her überflogen.

«Fantastisch!», flüsterte Luca ehrfurchtsvoll, während sein Vater nur geistesabwesend nickte. Der alte Mann schien mit seinen Gedanken schon weit über die Gebirgszüge hinaus in den Süden geschweift zu sein. Nach Montella, zu seinem jüngsten Bruder, den er morgen würde zu Grabe tragen müssen.

Heute Morgen erst hatte Luca den Anruf des Vaters erhalten; kurz vor zehn Uhr, mitten in einer Operation! Das Gespräch sei dringend, hatte es geheißen, genau wie damals vor acht Jahren, als sein Vater das letzte Mal in die Klinik angerufen hatte, um ihm mitzuteilen, dass seine Mutter gestorben sei. Und wieder war die Nachricht betrüblich gewesen. Allerdings musste er eingestehen, dass er erleichtert gewesen war, als er erfahren hatte, dass es um Carlo ging. "Nur Carlo», hatte er einen egoistischen Moment lang gedacht. Die plötzliche Angst um seine Frau Juliette und den fünfjährigen Domenico hatte sich als unbegründet erwiesen! Gott sei Dank! Aber dann hatte ihn doch ein Gefühl der Beklemmung ergriffen, und als sein Vater ihm völlig aufgelöst mitgeteilt hatte, dass er umgehend nach Montella reisen müsse, hatte Luca spontan angeboten, ihn zu begleiten. Juliette war mit dem Kleinen sowieso verreist, nach Montreal, zu ihren Eltern. Für ein paar Wochen, hatte sie gesagt, dauernd von einem «Time-out» gesprochen und dass sie Distanz brauche! ... Na ja, die hatte sie nun. Schon seit genau sieben Tagen und fünf Stunden! Und sie hatte sich noch kein einziges Mal gemeldet!

Zugegeben, in letzter Zeit hatten sie gewisse Probleme gehabt ... und dann das peinliche Intermezzo mit der Kardiologin! ... Eigentlich völlig harmlos, doch offensichtlich hatte Juliette das Ganze mehr zugesetzt, als er dachte. Aber dass sie deswegen gleich die Koffer packen musste?

Er seufzte tief, dann reckte er den Hals und ließ seinen Blick durch den Passagierraum schweifen, bis er einige Meter hinter sich die Flugbegleiterin entdeckte. Entspannt lächelnd versah die Frau ihren Dienst - wenigstens mit dem Flugzeug schien alles in Ordnung. Beruhigt schloss er die Augen.

Sein Vater, Salvatore Clemente, war ein wortkarger, verschlossener Mensch. Ein Gespräch mit ihm zu führen war nicht immer einfach, und oftmals erweckte er den Eindruck von Teilnahmslosigkeit oder Gleichgültigkeit. Nicht nur jetzt, im Moment der Trauer. Bis anhin hatte Luca es vermieden, den hageren, kleinen Mann auf Carlos Tod anzusprechen. Sie hatten über andere Dinge geredet, über das Angeln zum Beispiel oder über Fußball. Und über Motorräder. Belangloses Zeug eben, das ihm geeignet schien, seinen Vater nicht weiter in die Trübseligkeit abgleiten zu lassen. Aber früher oder später, dessen war er sich bewusst, würden sie trotzdem über das, was sie in Montella erwartete, sprechen müssen.

«Carlo soll schon morgen früh beigesetzt werden», griff Salvatore plötzlich das heikle Thema auf, als ob er die Gedanken seines Sohnes gelesen hätte! «Ich hoffe, dass alle rechtzeitig da sein werden.»

«Morgen? Wieso denn schon morgen?» «So ist es vorgeschrieben. In Montella müssen Beisetzungen spätestens vierundzwanzig Stunden nach Feststellung des Todes erfolgen. Außer an Sonntagen.»

\section{Rosas Blut - ein Kriminalroman bei EMH}

Mit «Rosas Blut» von Peter Hänni erweitert der Schweizerische Ärzteverlag EMH sein Buchprogramm um die Sparte Kriminalroman. Leserinnen und Leser der SÄZ können sich von den Qualitäten des Romans anhand einer Leseprobe überzeugen, die wir in vier aufeinanderfolgenden Ausgaben publizieren - und sie haben die Möglichkeit, das Buch direkt beim Verlag bestellen: online unter www.emh.ch/books oder über Tel. 0614678575 . Ein Artikel von Erhard Taverna zu Autor und Werk ist in der SÄZ Nr. 40/2008 erschienen.
«Warum findet sie überhaupt in Montella statt und nicht bei uns in der Schweiz?»

«Laura möchte es so! Die beiden hatten sowieso die Absicht, in zwei bis drei Jahren endgültig zurückzukehren und ihren Lebensabend dort zu verbringen. Ich nehme an, dass Laura jetzt alleine nach Montella ziehen wird und deshalb ihren Mann vor Ort begraben möchte.»

Salvatore Clemente hatte nie verstanden, wieso sein Bruder und seine Schwägerin nach über vierzig Jahren unbedingt nach Montella zurückkehren wollten. Natürlich war das Klima dort unten angenehmer und der Lebensunterhalt um einiges günstiger, aber er, Salvatore, hätte trotzdem nie zurückkehren können. Nicht wenn er seine Kinder und die Enkel in der Schweiz hätte zurücklassen müssen. Carlo und Laura hingegen hatten ihre Rückkehr schon seit langem geplant. Vor fünfzehn Jahren hatten sie ein Zweifamilienhaus gebaut, zusammen mit Lauras Bruder Aurelio und dessen Ehefrau Filomena. Filomena und Aurelio bewohnten in diesem Gebäude das Erdgeschoss, die Wohnung im Obergeschoss diente Carlo und Laura als Feriendomizil und hätte später zu ihrem neuen Heim werden sollen. Einige Jahre nach dem Bau des Hauses hatte Carlo auch ein altes, baufälliges Restaurant gekauft, das er im Laufe der Zeit praktisch eigenhändig renoviert hatte. Wahrscheinlich in der Hoffnung, dereinst auch seinen Sohn Francesco und dessen Familie zur Umsiedlung nach Montella bewegen zu können. Francesco war Koch und hatte eine Ausbildung als Gastwirt absolviert.

«Wie ist es eigentlich passiert?», fragte Luca.

Salvatore zögerte einen Moment, bevor er antwortete. «Filomena hat mir erzählt, Carlo habe in den letzten Tagen in den Kastanienwäldern gearbeitet, zusammen mit Francesco. Auch heute Morgen. Sie hat die beiden kurz vor sechs Uhr beim Verlassen des Hauses angetroffen. Carlo sei bester Laune gewesen und habe sich auf die Arbeit gefreut. Er habe in keiner Art und Weise über Beschwerden oder Unwohlsein geklagt. Im Wald angekommen, hätten sie offenbar zuerst die am Vortag gesammelten und in einer Hütte zwischengelagerten Kastanien in einen Klein- 
transporter verladen. Francesco sei anschließend zur Sammelstelle gefahren, um die Kastanien abzuliefern. Nach seiner Rückkehr habe er Carlo leblos am Boden gefunden, wenige Meter von der Hütte entfernt. Er habe sofort versucht, Wiederbelebungsmaßnahmen durchzuführen. Leider erfolglos!»

«Aber woran ist er eigentlich gestorben?», hakte Luca nach. «Herzversagen - jedenfalls nach Meinung des Arztes», antwortete Salvatore mit verächtlichem Unterton und fügte gereizt hinzu: «So ein Blödsinn! Carlo war noch jung und stark wie ein Stier! Er rauchte nicht, war kerngesund und hatte ganz gewiss nie Probleme mit dem Herz. In unserer Familie ist noch nie jemand an
Herzversagen gestorben. Nicht in dem Alter! Die Clementes sterben an Unfällen, an Krebs oder an Altersschwäche.

Früher, während des Krieges, vielleicht auch an Hunger oder Tuberkulose. Aber Herzversagen bei Carlo? Das kann ich nicht glauben!»

Luca versuchte gar nicht erst, seinem aufgewühlten Vater klarzumachen, dass ein neunundfünfzigjähriger Mann sehr wohl an plötzlichem Herzversagen sterben konnte - auch wenn im Vorfeld nichts auf ein solches Ende hingedeutet hatte. Stattdessen sagte er nur: «Ich kann ja morgen einmal mit diesem Arzt sprechen, von Kollege zu Kollege. Vielleicht wissen wir danach mehr.»
«Ja! Genau das wirst du tun!», pflichtete Salvatore in verschwörerischem Ton bei und tätschelte seinem Sohn den Oberschenkel. «Das ist eine sehr gute Idee!» Dann fügte er hinzu: «Ich bin froh, dass du mich begleitest, mein Junge! Und jetzt möchte ich mich noch ein wenig ausruhen.»

Er knüllte seinen Pullover umständlich zu einer Art Kissen zusammen, lehnte sich seitlich ans Fenster und verschränkte die Arme vor der Brust. Dann schloss er die Augen. Luca betrachtete seinen Vater einen Augenblick lang schweigend, dann stellte er seinen Sitz zurück, streckte die Beine zum Mittelgang hin aus und schloss ebenfalls die Augen.

\section{Wie die Moderne aus der Grube kam}

\section{Erhard Taverna}

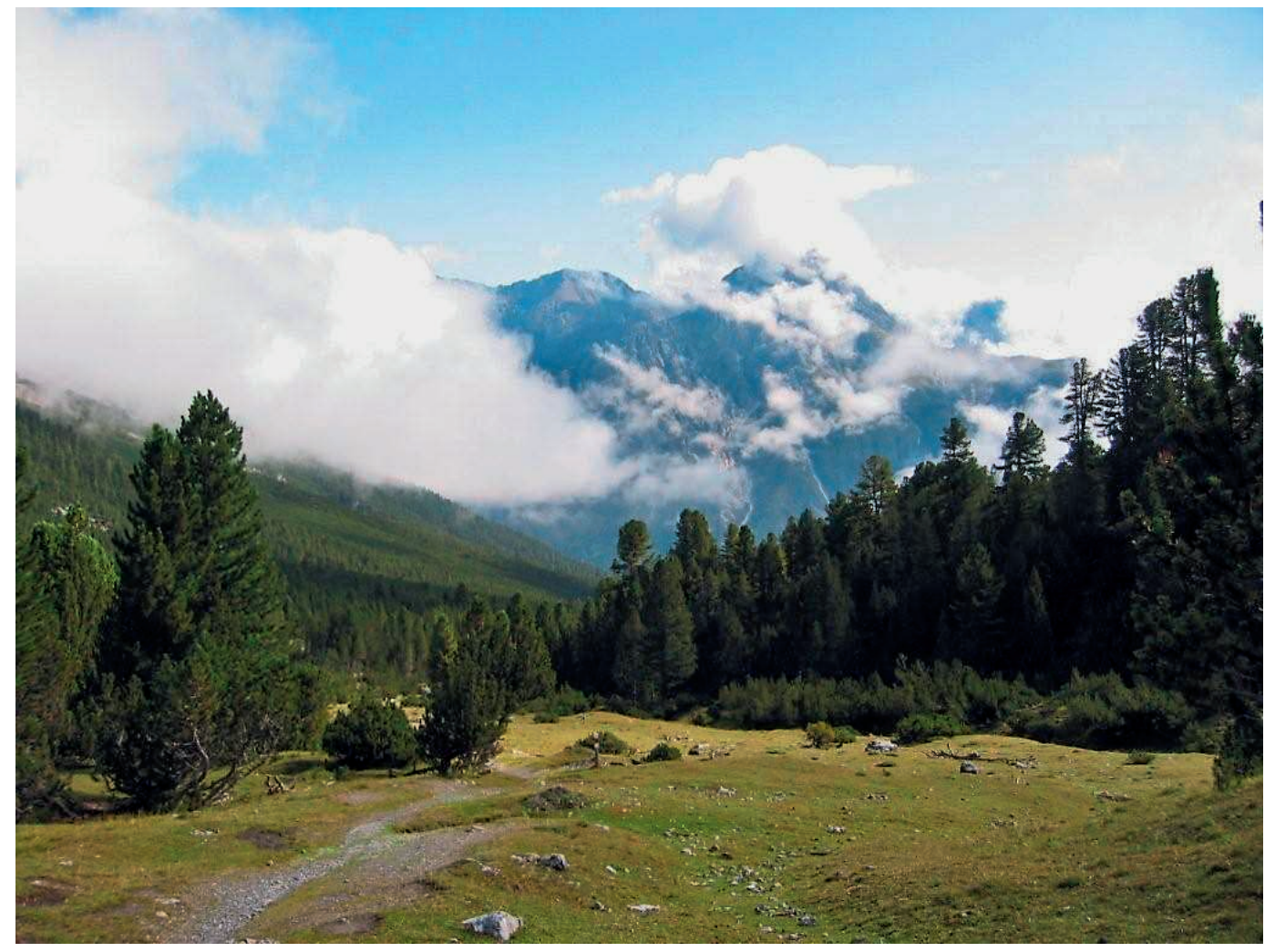

Sommer und Herbst sind Wanderzeit. Was gibt es Erholsameres, als über die alten Passstrassen der Säumer und auf einsamen Wegen im Nationalpark zu wandern, dem melancholischen Ruf der cratschla (Tannenhäher) oder dem Wind in den Bäumen zu lauschen? In den Lärchenwäldern am Ofenpass, zwischen Arven in Tamangur oder Legföhren im Val Mingèr, beim Beobachten der Hirschkühe und Murmeltiere scheint die Illusion der sich selbst überlassenen Natur perfekt. Nur einige Flurnamen und wenige Ruinen erinnern an die frühere Industriezone am Rande der nahe gelegenen habsburgischen Bergbauzentren in Schwatz und Hall. Es sind Namen wie Fuorn für Schmelzofen, Mingèr für Knappe, Sesvenna für Erzstollen oder Taglieda für Kahlschlag. Das Bergbaumuseum Schmelzra in S-charl bietet eine fesselnde und lehrreiche Einführung in eine Industriekultur, die wegen der mythischen Identifikation mit einer bäuerlichen Vergangenheit gerne 
verdrängt wird [1]. Die Ausstellung erinnert unter dem Motto «Die Schweiz ist reich an armen Gruben» an die jahrhundertealte, vielfältige Rohstoffgewinnung im ganzen Alpenraum als Grundlage des modernen Industriestaates.

Wo sich heute Feriengäste verwöhnen lassen, hauste der aus dem Tirol eingesetzte Bergrichter über die streitbare Belegschaft der «wilden Insel», wie die Siedlung im Unterengadin in einer bischöflichen Chronik genannt wurde. Erzhaltiges Gestein wurde in härtester Handarbeit aus den zahlreichen Stollen am Muot Madlain herausgepickelt, zerkleinert und eingeschmolzen; aus drei Tonnen drei Kilogramm Blei und dreissig Gramm Silber gewonnen und nach Innsbruck geliefert. Der mittelalterliche Bergbau hat die Entwicklung von Chemie und Medizin vorangetrieben, denn die Umwandlung der Verbindungen, Metallschmelze und Bestimmung des Metallgehaltes stehen im Zentrum der Alchemie. Neue Metalle wie Zink, Wismut, Kobalt (nach dem Berggeist Kobold) und Kupfernickel erweiterten die Liste der antiken Vorgänger.

Nur am Rande werden zwei berühmte Ärzte erwähnt, deren Schicksal wesentlich durch den Bergbau bestimmt wurde. Der eine ist Paracelsus (1493-1541), der andere Georgius Agricola, mit bürgerlichem Namen Georg Bauer (1494-1555). Paracelsus ist als Alchemist, Astrologe, Mystiker und Philosoph unter Medizinern bekannter als der Stadtarzt und Bürgermeister in Chemnitz, der mit seinen Werken die Geowissenschaften begründete. Paracelsus schreibt über Bergbaukrankheiten und ersetzt die antike Säftelehre durch seine alchemistische Therapietriade aus Schwefel, Quecksilber und Salz. Agricola geht in seinem Standardwerk «De re metallica libri XII» ebenfalls auf montane Krankheiten ein, ist aber alchemiekritischer und technologischer eingestellt. Der Erste hat die Medizin revolutioniert, der Zweite hat in seinen Schriften den wissenschaftlichen Rationalismus vorausgedacht. Beide sind herausragende Pioniere, die dem Aberglauben ihrer Zeit verhaftet bleiben. Paracelsus ist von der Macht der Gestirne überzeugt, Agricola, der eine massgebliche Theorie zur Entstehung von Metalladern entwickelt, beschreibt Kobolde und Drachen im Erdinnern. Mit Agricola wird der Bergbau, neben dem Schiffsbau und der Kriegstechnik, zum primären Übungsfeld unseres Umganges mit der Natur. Die Technik ist für ihn wertneutral, und die alte Naturphilosophie wird verabschiedet, denn ausser der menschlichen Zweckordnung erkennt er keine andere Ordnung der Natur. Die Erde ist ein Aggregat von Materie, das der zivilisierte Mensch durch seine Anstrengungen veredelt und einer höheren Bestimmung zuführt. Agricola argumentiert wie die meisten Ökonomen und Technokraten gegenüber heutigen Naturschützern, indem er geschickt die damaligen Kritiker des Bergbaus als Ignoranten und Fortschrittsfeinde hinstellt. Ob Abholzen der Wälder mit Energie- und Wassermangel als Folge, ob Lebensmittelknappheit und Teuerung, ob Habgier und schlechte Arbeitsbedingungen oder Sklaverei und Genozid in der neuen Welt, die Gegner der Metallgewinnung sind prinzipielle Fortschrittsfeinde. Wer nicht für den Bergbau ist, hemmt die gottgewollte Selbstentfaltung der Menschheit.

Die Spitzhacken und Pochwerke haben im S-charl-Tal ausgedient. Köhler und Flösser sind weggezogen, über die alten Narben ist der neue Wald gewachsen, Hirsch und Steinbock sind zurückgekehrt. Nur Mangel oder Geld könnten die alten Stollen wiederbeleben, wie in den 1940er Jahren, als im Gonzen wieder Eisen und im Oberhalbstein Mangan abgebaut wurde. Auch das vormoderne Wissen von der Lebendigkeit der Natur schlummert weiter in der Tiefe. Denn Alchemie und Bergwerk leben als Archive der Erinnerung in unserem Unbewussten, sie inspirierten Märchen und Sagen und die Romantiker, von Novalis' «Heinrich von Ofterdingen» über Hoffmanns «Die Bergwerke zu Falun» bis zum Klassiker der Fantasyliteratur, Tolkiens «Herr der Ringe». Das Zensierte und Marginalisierte belebt die okkulten Lehren der Neuzeit, die Psychoanalyse, die Phantasmen und Obsessionen, den Wahnsinn und die Kunst, die Utopien und Gegenutopien, die Sehnsüchte und Alpträume der sogenannten Wissensgesellschaft. 